\title{
Spotting Brain and Pancreatic Tumor using Fuzzy C-Mean Segmentation and SIFT Extraction Through Sparse Representation Method
}

\author{
Jithendra Reddy. D, Arun Prasath. T, Pallikonda Rajasekaran. M, Vishnuvarthanan. G
}

\begin{abstract}
Diagnosis of Neoplasm is an utmost recurrent and lethal technique for detecting a malignant primary tumor. Imaging techniques empower researchers and medical practitioners to evaluate disorders and activities inside the human brain earlier than performing invasive surgery. Here presents the spotting and detection of brain tumor and pancreatic tumor segmentation and classification progression with several stages DBCWMF algorithm filter with histogram equation, Precise Fuzzy C-segmentation, and SIFT extraction and classification with Sparse representation. These techniques provide a better ability in clinical practices in terms of speed, accuracy, innovation. Experimental results were evaluated using TCIA database and hospital database, where the proposed approaches were verified simultaneously with data progression and incredibly effective for brain and pancreatic tumor in MR images and CT scan images both.
\end{abstract}

Keywords : Brain tumor, DBCWMF, Fuzzy C-segmentation, SIFT, Sparse representation, pancreatic tumor.

\section{INTRODUCTION}

Soft computing is immensely exerted in medical scrutiny medical image processing. MRI scans are exerted to create images all body parts and it dispenses an effective and rapid way for analysis of the brain tumor and pancreatic tumor exerted as an important tool while doing surgical and clinical atmosphere for the reason that of its characteristics similarly greater soft tissue diversity, higher spatial determination and with contrast significant analytical imaging method to get premature identification the brain tumor [1]. Brain imaging of MRI shows a vital key part in succor radiologists has to be approached patients for analysis and remedy [2]. The utmost eminent significant of medical image perusal in general, brain magnetic resonance image (MRI) examination in particular, is to extract clinical data that help to revamp diagnosis and remedy of ailment.

Revised Manuscript Received on December 05, 2019.

* Correspondence Author

D. Jithendra Reddy*, Department of Instrumentation and Control Engineering, Kalasalingam Academy of Research and Education, Virudhunagar, India. Email: jithendrareddy.d@gmail.com

T. Arun Prasath, Department of Biomedical Engineering, Kalasalingam Academy of Research and Education, Virudhunagar, India. Email: arun.aklu@gmail.com

M. Pallikonda Rajasekaran, Department of Electronics and

Communication Engineering, Kalasalingam Academy of Research and

Education, Virudhunagar, India. Email: mpraja80@gmail.com

G. Vishnuvarthanan, Department of Biomedical Engineering,

Kalasalingam Academy of Research and Education, Virudhunagar, India. Email: gvvarthanan@gmail.com
Brain tumors are one of the utmost recurrent brain ailments, so the classification of brain tumors in MRI is essential in medical diagnosis [3].

\section{RELATED WORK}

With CNN bounding and elective scrawls as user interactions and user afford images which are tested, nonetheless they might possibly be gained by involuntary detection [36] to auxiliary growth in efficiency Guotai, et al. [4]. The Noise reduction has made a stage in preprocessing imaging classification as stated by Sebe et al. Here the author has been analyzed exert Quadrature Mirror Filter and Gabor uses for noise reduction purposes has given emphasis to the need for preprocessing. The method is needed in order to eliminate artifacts delay additional processing of MRI images. Pre-processing images stay essential for analysis in Computer-Aided Diagnosis. The author exerted a square designed to structure element and median filtering [5] exerted the elimination of artifacts in a preprocessing stage. It will Creates sure set 255 as a threshold value of aimed at artifacts elimination and 200 as its threshold values for the elimination of the undesirable a part of a whole of the image [6]. Author Ratan has exerted an altered type of preprocessing for decreasing the processing volume of data. Total 128 images were processed into 3 clips [7]. In Mohan et al. article, here analyzed sundry articles that ensured handle image preprocessing methods. He states that although sundry methods to eliminate noise-introducing negative result on the segmented part of the image [8]. Texture features were associations with bidirectional of associative memory category on artificial neural network is to be classifying the soft tissue parts in brain CT images Sharma,. It was combined with edge features and gray level classified using SVM for brain CT images Padma Nanthagopal, [9]. Combining of texture features with Naive Bayesian classifier from benign tumor and malignant tumor images. Kharrat combining of texture features with SVM classifier is to classify those normal tumors, benign tumors and malignant tumors images. Padma combined coexistence texture features with (PNN) probabilistic neural network classifier used to classify human brain CT scan images [10]. This paper explains about 4 Stages first conversions of noise MRI images to filtered DBCWMF images. 
After Conversion of a filtered image the second step is a detection of brain and pancreatic tumor part Fuzzy Csegmentation is carried out through Sparse Representation Classifier. Involuntary segmentation of multi-atlas process now broadly used for brain imaging.

reference section. In the case of exclusion of references, it should be less than $5 \%$.

\section{PROPOSED METHODOLOGY}

The methodology proposed involves the Decision Based Couple Window Median Filter, histogram equation, Fuzzy C-segmentation SIFT extraction and classification with Sparse representation (Fig. 1).

Essential phases involved the proposed Methodology:

i) MR image and CT scanned Medical image input

ii) Preprocessing: DBCWMF image in filter and histogram equation

iii) Segmentation: Fuzzy C- segmentation with identifying Neoplasm

iv) Feature extraction: SIFT image extraction

vi) Classification: Sparse representation of accuracy.

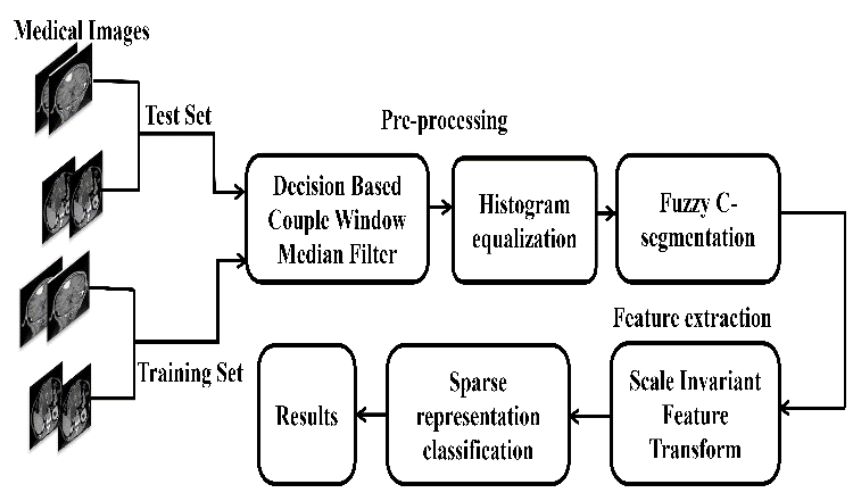

Fig.1. Block diagram proposed

\section{A. Decision Based Couple Window Median Filter algorithm}

After median filter imaging leftover noise pixels free be a selection of by the window median filter with changing pixels of boisterous [11]. The method from the first image noise pixel and ultimately the pixel of a noise image ends. The scanned caused image entitled as a restored image. The mainly 3/4th or grater pixels noisy are transformed into pixels' noise-free with a median filter. Except affected pixels left over free pixel noise picks the window as pixels (i.e. 0 and 255).

$$
\begin{array}{r}
0<\mathrm{A}(, \mathrm{n})<255, \text { Noise free } \\
\mathrm{X}(\mathrm{y}, \mathrm{z})=\mathrm{Y}(\mathrm{y}, \mathrm{z})
\end{array}
$$

$$
M=(2 n+1) a(2 n+1)
$$

$$
\mathrm{M}(\mathrm{y}, \mathrm{z})=\operatorname{mean}\{\mathrm{Z}(\mathrm{y}, \mathrm{z})\}
$$

\section{B. Histogram equalization}

Histogram equalization progresses the areas of the pixels that are overexposed and underexposed production the pixies histogram unchanging. Before and after histogram equation pixels find the middle element in the window compute the function of cumulative distribution of the values in the window [12].

$$
\operatorname{cdf}(1)=\operatorname{cdf}(1)+\operatorname{cdf}(1-1)
$$

$$
I(i, j)=\operatorname{round}(c d f(e l e)(A * B) * 255)
$$

\section{Segmentation}

Fuzzy C mean understand the imaging; a suitable segment is essential. Foremost task is to fit clusters with into 1 or more. The collected function is assumed as [13].

$L_{n}=\sum_{a=1}^{N} \sum_{b=1}^{C} v_{a b}^{r}\left\|x_{a}-c_{b}\right\|^{2}$

$1 \leq \mathrm{r}<\infty$

Where,

$r$ is real number $<1$;

$v_{a b}$ is the degree of $x_{a}$ members of in the $l$ cluster;

$x_{a}$ is the d-dimensional $a^{\text {th }}$ of data;

$c_{b}$ is the center of the cluster d-dimension;

\section{Feature Extraction SIFT}

This is used to achieve the image feature extraction. This performance is eventually used for matching image [14]. An SIFT improvement with the comparative method of measuring the entire response belongs to the neighbor's interest point [15]. Key point identifiers can be massively repeatable.

$$
\mathrm{A}(\mathrm{x}, \mathrm{y}, \sigma)=(\mathrm{B}(\mathrm{x}, \mathrm{y}, \mathrm{k} \sigma)-\mathrm{G}(\mathrm{x}, \mathrm{y}, \sigma) \times \mathrm{C}(\mathrm{x}, \mathrm{y}))
$$

\section{E. Screening Sparse representation classification}

[16-17]. The principal aim of SR remains input signal linear combination done SR vector and ample dictionary. The key problems in SR are how to construct and solve vector done with ample dictionary. Widespread tools to build the dictionary contains the method of elective directions (K-SVD) k-singular value a decomposition online learning and algorithm discrete cosine transform. This effectually computed through optimization or greedy methods.

The training data matrix $\mathrm{A}$, the known label vector $\mathrm{B}$, and $\mathrm{x}$ observation.

SR Screening: Calculate

$\Omega=$ fат1 а; ат2 a; · · · ;atnag

$\mathrm{T}=$ transpose), $. A x=f a(1) ; a(2) ;::: a(z) g$ with

$$
\mathrm{z}=\min \mathrm{fn}=\log (\mathrm{c}) ; \mathrm{mg}
$$

where aT (i)a is the ith major element $\Omega$.

Regression: Crack the normal problem of least square between $\mathrm{Ab}$ and $\mathrm{a}$. Namely, compute $\beta=a b-1 a$

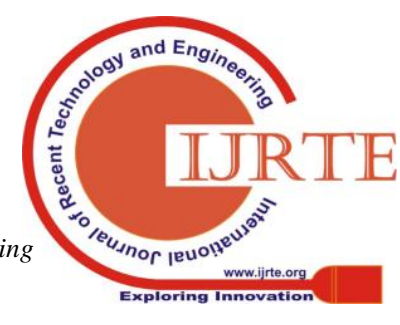


where $a b-1$ is the Moore-Penrose inverse.

SR Classification: Allocate the testing thought by $\operatorname{gnscr}(\mathrm{a})=\arg \min y 2[\mathrm{Y}]$

$$
\theta\left(\mathrm{a} ; \operatorname{Aby}^{\wedge} \mathrm{y}\right)
$$

Where $\theta$ denotes the principal angle. Break ties deterministically.

SR Output: The estimated class label gnscr (a)

\section{RESULTS}

Experimental carry out with $512 \times 512$ brain and $256 \times 256$ pancreases images 30 to 60 degrees respectively via MATLAB 2013aCT scan and MR images are used. Fragmented 100 images as 40 for testing and 60 for training by TCIA database and hospital database [18].
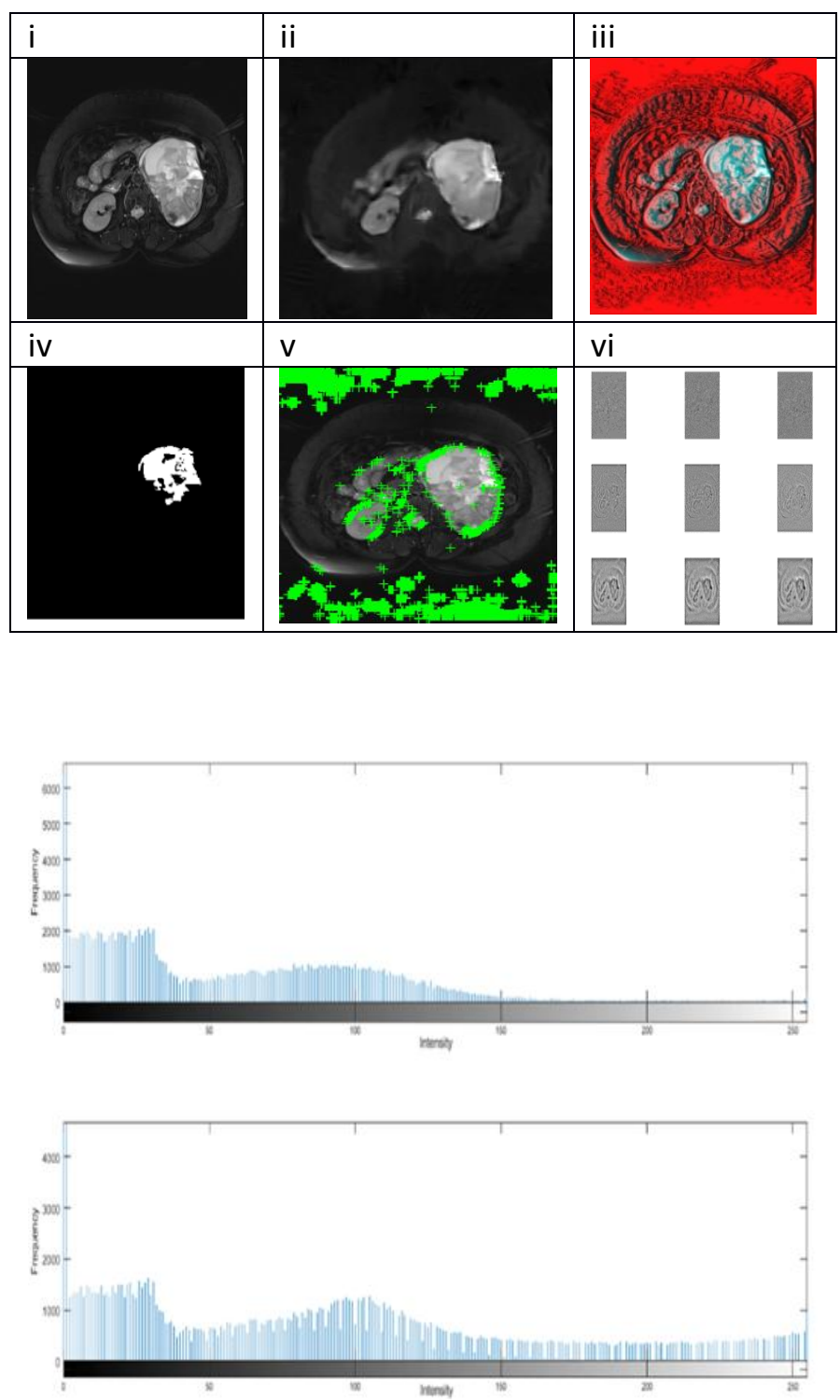

Fig.2. (i) Brain input (ii) Prepressing (iii) Histogram output (iv)Segmentation (v) \&(vi) Feature extraction(vii) Histogram graph

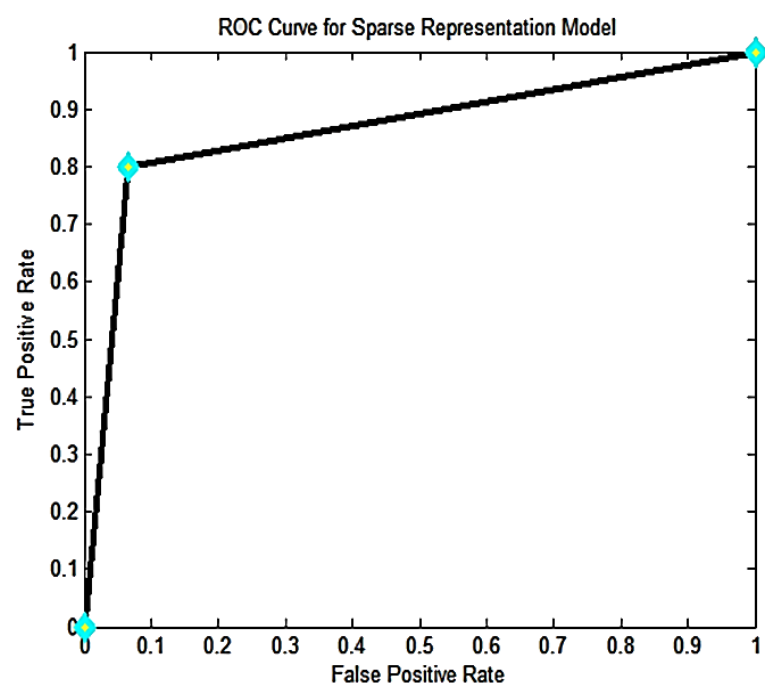

Fig.3. ROC curve -Brain

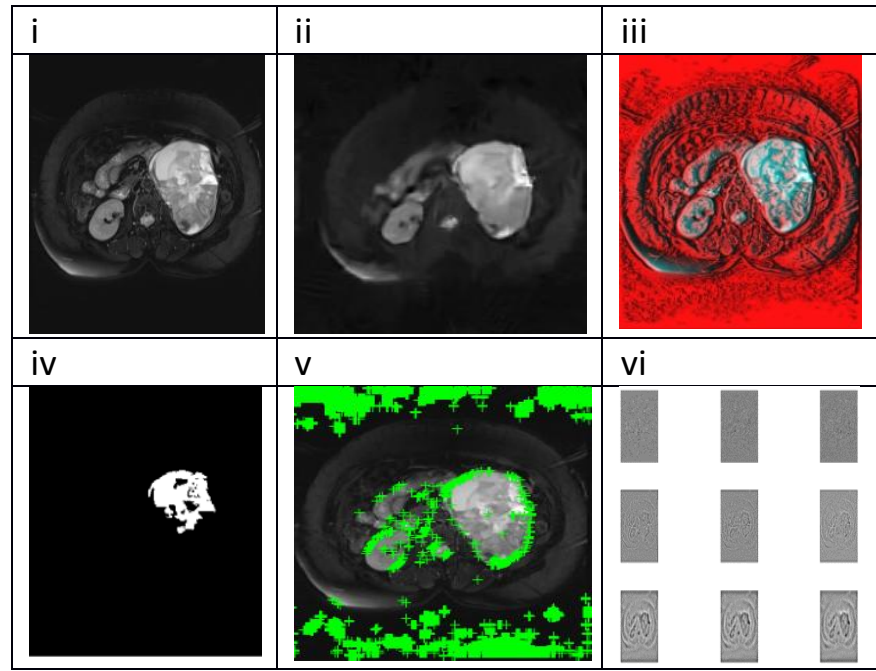

Fig.4. (i) Pancreas input (ii) Prepressing (iii)Histogram output (iv) Segmentation (v) \&(vi) Feature extraction (vii) Histogram graph 

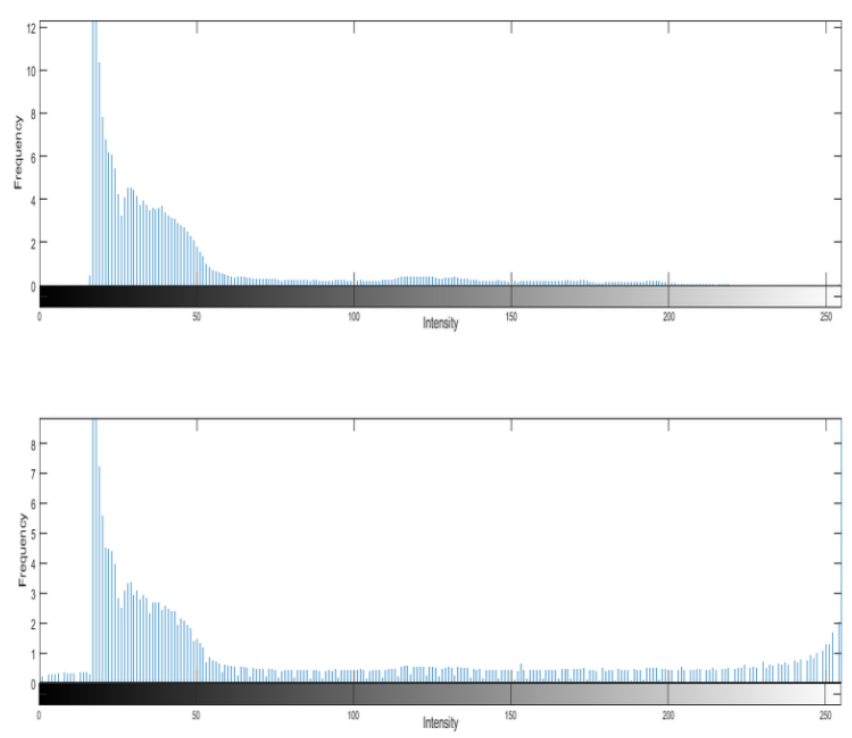

Fig.4. (i) Pancreas input (ii) Prepressing (iii)Histogram output (iv) Segmentation (v) \&(vi) Feature extraction (vii) Histogram graph

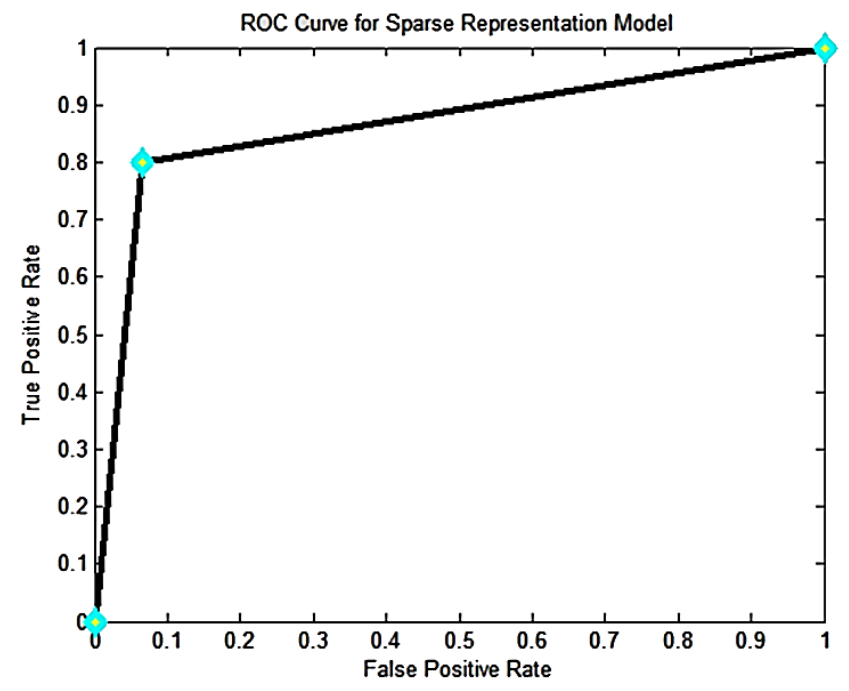

Fig.5. ROC curve -pancreases

TABLE I. Brain-parameters

\begin{tabular}{|l|l|l|l|l|}
\hline Parameters & MSE & PSNR & SSIM & NCC \\
\hline Median & 230.22 & 21.41 & 0.4021 & 1 \\
\hline DBCWMF & 101.51 & 28.87 & 0.7735 & 1 \\
\hline
\end{tabular}

TABLE II. Pancreatic-parameters

\begin{tabular}{|l|l|l|l|l|}
\hline Parameters & MSE & PSNR & SSIM & NCC \\
\hline Median & 176.23 & 26.16 & 0.6123 & 1 \\
\hline DBCWMF & 88.34 & 30.27 & 0.8267 & 1 \\
\hline
\end{tabular}

$$
\operatorname{MSE}=\left[\frac{1}{P Q}\right] \sum_{c=0}^{P-1} \sum_{d=0}^{Q-1}[F(c, d)-\hat{F}(c, d)]^{2}
$$

$$
\begin{aligned}
P S N R=10 \log _{10} \frac{255}{M S E} \\
N C C=\frac{\sum_{y=1}^{c} \sum_{z=1}^{d}\left(P_{y z} * Q_{y z}\right)}{\sum_{y=1}^{c} \sum_{z=1}^{d}\left(P_{y z}\right)}
\end{aligned}
$$

\begin{tabular}{|c|c|c|}
\hline & Brain & Pancreatic \\
\hline Accuracy & $93.67 \%$ & $89.21 \%$ \\
\hline Precision & $97 \%$ & $90.86 \%$ \\
\hline Specificity & $97 \%$ & $91.26 \%$ \\
\hline Recall & $91.24 \%$ & $84.67 \%$ \\
\hline
\end{tabular}

TABLE III. Performance Analysis

$$
\begin{array}{lr}
T P & \text { Precision }= \\
F P+T P & (14)
\end{array}
$$

$$
\text { Recall }=\frac{T P}{F N+T P}
$$

TABLE IV. Accuracy Analysis with different Models-Brain

\begin{tabular}{|l|l|l|l|}
\hline & $\begin{array}{l}\text { Probability } \\
\text { neural } \\
\text { networks } \\
\text { (PNN) }\end{array}$ & ANN & $\begin{array}{l}\text { Sparse } \\
\text { Representation }\end{array}$ \\
\hline Accuracy & $83.33 \%$ & 82.24 & $93.67 \%$ \\
\hline
\end{tabular}

TABLE V. Accuracy Analysis with different modelsPancreatic

\begin{tabular}{|l|l|l|l|}
\hline & $\begin{array}{l}\text { Probability } \\
\text { neural } \\
\text { networks } \\
\text { (PNN) }\end{array}$ & ANN & $\begin{array}{l}\text { Sparse } \\
\text { Representation }\end{array}$ \\
\hline Accuracy & $80.33 \%$ & $82.24 \%$ & $91.21 \%$ \\
\hline
\end{tabular}




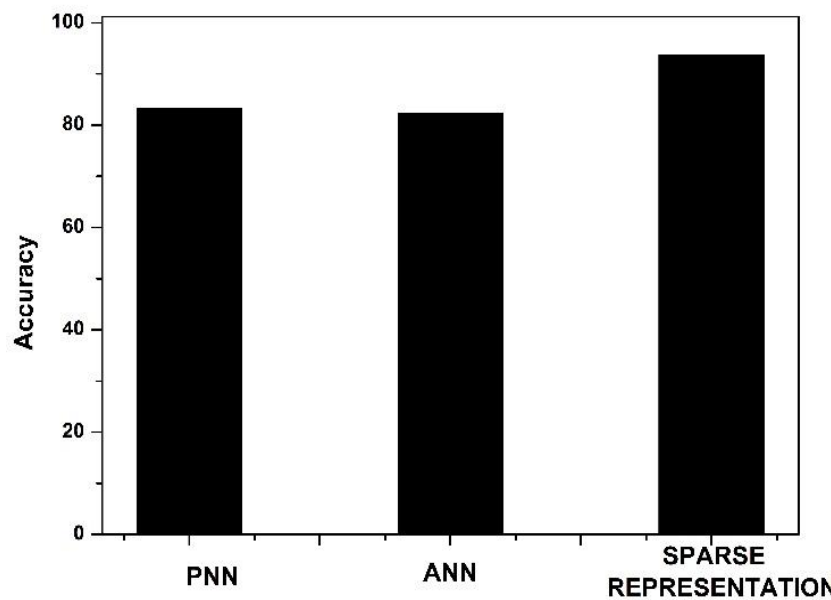

Fig.6. Accuracy Analysis with different Models-Brain

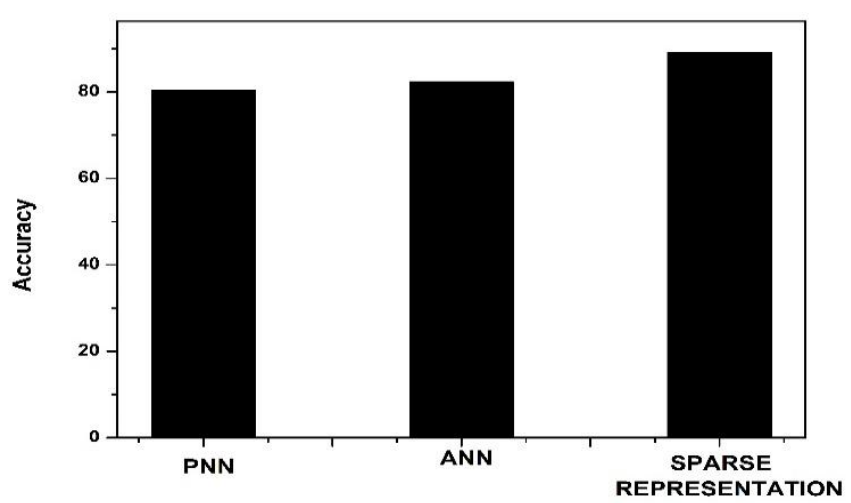

Fig.7. Accuracy Analysis with different models- Pancreatic

\section{CONCLUSION}

Imaging technology has had rapid growth in imaging field. The proposed methodology represents spotting Tumor on both pancreatic CT scan and brain MR images filtered using DBCWMF, which is higher than median filter and Histogram equation. The segmentation with Fuzzy C-mean algorithm and the classification is done by employing Screening Sparse representation and has higher results comparing with ANN and PNN.

\section{ACKNOWLEDGMENT}

The Author is thankful to the management of Kalasalingam Academy of Research and Education for providing fellowship and also thanks VLSI DST-FIST Research Centre for supporting laboratory facilities during this research work. The author is thankful to Dr. Daggumati Shrehari Rao, Radiologist, Chairman \& Administrative Committee Member of Andhra Pradesh Medical Council Vijayawada (Indian Government) (Chairman \& Managing Director of Sri Sai Seven Hills Multispecialty Hospitals Tirupati) providing necessary patient information and images for supporting research.

\section{REFERENCES}

1. Drozdzal, Michal, et al. "Learning normalized inputs for iterative estimation in medical image segmentation." Medical image analysis 44 (2018): 1-13.

2. Wang, Guotai, et al. "Interactive medical image segmentation using deep learning with image-specific fine tuning." IEEE transactions on medical imaging 37.7 (2018): 1562-1573.

3. Roth HR, Lu L, Farag A, Shin H-C, Liu J, Turkbey EB, Summers RM. DeepOrgan: Multi-level Deep Convolutional Networks for Automated Pancreas Segmentation. N. Navab et al. (Eds.): MICCAI 2015, Part I, LNCS 9349, pp. 556-564, 2015.

4. Wang, Guotai, et al. "Interactive medical image segmentation using deep learning with image-specific fine tuning." IEEE transactions on medical imaging 37.7 (2018): 1562-1573.

5. Sonali Patil, V. R. Udupi "International Journal of Computer Science Engineering and Information Technology Research" ISSN 2249-6831 Vol. 3, Issue 1, Mar 2013, 61-66.

6. Wang, Guotai, et al. "DeepIGeoS: a deep interactive geodesic framework for medical image segmentation." IEEE transactions on pattern analysis and machine intelligence (2018)

7. Rajinikanth, V., et al. "Entropy based segmentation of tumor from brain MR images-a study with teaching learning based optimization." Pattern Recognition Letters 94 (2017): 87-95.

8. Mohan, Geethu, and M. Monica Subashini. "MRI based medical image analysis: Survey on brain tumor grade classification." Biomedical Signal Processing and Control 39 (2018): 139-161.

9. Padma Nanthagopal A \& Sukanesh, R 2013, 'Wavelet statistical texture features-based segmentation and classification of brain computed tomography images' IET Image Processinging, vol. 7, no. 1, pp. 25-32.

10. Virmani, Jitendra, Nilanjan Dey, and Vinod Kumar. "PCA-PNN and PCA-SVM based CAD systems for breast density classification." Applications of intelligent optimization in biology and medicine. Springer, Cham, 2016. 159-180.

11. Dandu, Jithendra Reddy, et al. "Brain and pancreatic tumor segmentation using SRM and BPNN classification." Health and Technology (2019): 1-9.

12. LeeWT,tzong Chen H (2009) Histogram-based interest point detectors. In: Proceedings of the IEEE Computer Society Conference on Computer Vision and Pattern Recognition, pp 1590-1596

13. Reddy, D. Jithendra, et al. "Brain and Pancreatic Tumor Classification Based on GLCM-k-NN Approaches." International Conference on Intelligent Computing and Applications. Springer, Singapore, 2019

14. He, Yi, et al. "Optimization of SIFT algorithm for fast-image feature extraction in line-scanning ophthalmoscope." Optik 152 (2018): 2128.

15. Li, Qingliang, et al. "A Local Neighborhood Constraint Method for SIFT Features Matching." Recent Developments in Data Science and Business Analytics. Springer, Cham, 2018. 313-320.

16. Rigamonti, R.; Brown, M.A.; Lepetit, V. Are sparse representations really relevant for image classification? In Proceedings of the IEEE Conference on Computer Vision \& Pattern Recognition, Colorado Springs, CO, USA, 20-25 June 2011.

17. Peng, Y.; Ganesh, A.; Wright, J.; Xu, W.; Ma, Y. RASL: Robust alignment by sparse and low-rank decomposition for linearly correlated images. IEEE Trans. Pattern Anal. Mach. Intell. 2011, 34 , 2233-2246.

18. Holger R. Roth, Amal Farag, Evrim B. Turkbey, Le Lu, Jiamin Liu, and Ronald M. Summers. (2016). Data From Pancreas-CT.The Cancer Imaging Archive. http://doi.org/10.7937/K9/TCIA.2016.tNB1kqBU 


\section{AUTHORS PROFILE}

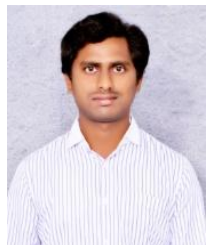

D. Jithendra Reddy Research Scholar in the Department of Instrumentation and Control Engineering at Kalasalingam Academy of Research and Education. He perused his Master of Technology in Digital Systems and Computer Electronics from JNTU Ananthapur University in 2015 and B.Tech in Electronics and Instrumentation Engineering from JNTU Ananthapur University 2011. His areas of interest are Medical Image processing.

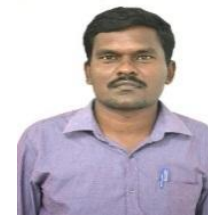

T. Arun prasath Associate Professor in Department of Biomedical Engineering at Kalasalingam Academy of Research and Education. He received Ph.D. in Electronics and Communication Engineering from Kalasalingam University in 2015, his M.E. in Applied

Electronics from Anna University in 2009 and his B.E. in Electrical and Electronics Engineering from Anna University in 2006. His research interest is image processing.

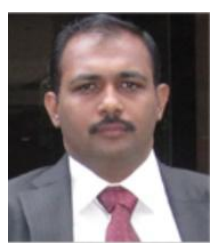

M. Pallikonda Rajasekaran Professor in School of Electronics and Electrical Technology at Kalasalingam University. He received his Ph.D. in Electrical and Electronics Engineering from Anna University, his M.Tech. in Biomedical Signal Processing and Instrumentation from SASTRA University in 2002 and his B.E. in Electronics and Instrumentation Engineering from Bharathidasan University in 2001 His research interest is medical image processing.

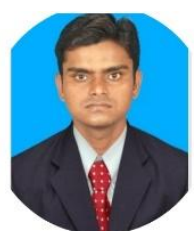

G. Vishnuvarthanan Associate Professor in Biomedical Engineering in Kalasalingam Academy of Research and Education. He has completed B.E. in Instrumentation and Control Engineering in 2007 from Anna University, and M.Tech. in 2009 from Bharath University. He holds a Ph.D. in Department of Electronics and Communication Engineering from Kalasalingam University, $\mathrm{His}$ research interest is medical image processing. 